\title{
Electronic Structure of Boron Doped HOPG: Selective Observation of Carbon and Trace Dope Boron by Means of X-ray Emission and Absorption Spectroscopy*
}

\author{
Masahito Niibe, ${ }^{\dagger}$ Noritaka Takehira, and Takashi Tokushima \\ Laboratory of Advanced Science and Technology for Industry, \\ University of Hyogo, Kouto 3-1-2, Kamigori, Ako-gun, Hyogo 678-1205, Japan
}

(Received 5 January 2018; Accepted 26 March 2018; Published 2 May 2018)

\begin{abstract}
We have measured the soft X-ray absorption and emission spectra of near B-K and C-K absorption edges of highly oriented pyrolytic graphite (HOPG) doped with a small amount (0.32 at.\%) of boron (B), including incident/takeoff angle dependence. The main peak intensities at the B-K edge around $182 \mathrm{eV}$ were almost independent on the change of takeoff angle. On the other hand, the satellite peak around $185 \mathrm{eV}$ becomes larger as the takeoff angle becomes smaller. Based on this result, unlike the previous assignment of the main peak predicted from the quantum chemical calculation ( $\pi$ orbitals), it is reasonable to attribute the main peak to the $\sigma$ orbitals. The takeoff angle dependence of the B-K emission is similar to the angle dependency of the C-K emission of undoped HOPG. Based on this and the theoretical consideration, it is presumable that the doped $\mathrm{B}$ atom does not intercalate between the layers of HOPG; it replaces the $\mathrm{C}$ atom of the hexagonal network structure and has a wave function similar to that of $\mathrm{C}$ atoms ( $\mathrm{sp}^{2}$ type hybridized orbital). [DOI: 10.1380/ejssnt.2018.122]
\end{abstract}

Keywords: Soft X-ray; B-K absorption edge; Incident/takeoff angle dependence

\section{INTRODUCTION}

The physical and chemical properties of graphite and graphene can be changed relatively easily by doping. Therefore, these materials are expected to be applied to various devices such as superconductors, catalysts, sensors, transistors, and positive-electrode materials of $\mathrm{Li}$ ion batteries. Understanding and controlling the electronic properties of these substances, especially the local electronic structure in the vicinity of the dopant, are indispensable for promoting the application for the various devices mentioned above. Soft X-ray absorption and emission spectroscopy are extremely effective methods for investigating the local electronic structure around the atom of interest in matter $[1,2]$.

The solubility of $\mathrm{B}$ in graphite at equilibrium is very low (less than a few percent) [3]. Furthermore, in many research reports such as lattice constant measurement by XRD [3], decrease in local lattice symmetry measured by Raman spectroscopy [4], and deformation of graphite planes observed by STM image [5], doped B atoms replace $\mathrm{C}$ atoms and are considered to enter substitution sites of the carbon hexagonal network structure. However, direct observation of change in electronic structure measured by $\mathrm{X}$-ray emission and absorption spectroscopy including polarization and incident/takeoff angle dependency provide very important information which cannot obtained by the above mentioned methods.

Soft X-ray emission efficiency from the B atom is very low [6]; therefore, it is difficult to observe the soft $\mathrm{X}$ ray emission from boron dissolved in a trace amount in graphite. In a previous study, Hanafusa et al. reported $\mathrm{X}$-ray emission and absorption spectra on B-K and C-K edge for a B-doped highly oriented graphite thin film (BHOGF) synthesized at a high temperature with estimated $\mathrm{B}$ concentration around 0.4 to $2.2 \%$ [7]. They have also

\footnotetext{
* This paper was presented at the 8th International Symposium on Surface Science, Tsukuba International Congress Center, Tsukuba, Japan, October 22-26, 2017.

† Corresponding author: niibe@lasti.u-hyogo.ac.jp
}

reported incident angle dependence for B-K and $\mathrm{C}-\mathrm{K}$ for X-ray absorption spectra.

We investigated boron-doped highly oriented pyrolytic graphite (HOPG) with boron concentration of $0.32 \%$, which is much lower concentration than the previous report, by means of soft X-ray absorption and emission spectroscopy for $\mathrm{B}-\mathrm{K}$ and $\mathrm{C}-\mathrm{K}$ edges including changes in incident and takeoff angles. We found evident takeoff angle dependence for B-K emission spectra of borondoped HOPG which was not reported in previous reports. Evident angle dependence for B-K emission is a direct evidence that doped $\mathrm{B}$ atoms replace $\mathrm{C}$ atoms in the carbon hexagonal network of HOPG.

\section{EXPERIMENT}

The sample is commercially available boron-doped HOPG (Union Carbide, ZYC grade). Composition analysis at the Japan Atomic Energy Research Institute (JAERI) revealed that the boron concentration was 0.32 at.\% [8]. The B doping amount was a trace amount, and almost no signal of the B 1s level could be detected by measurement with a commercially available X-ray photoelectron spectroscopy (XPS) apparatus (Shimadzu ESCA-1000). Soft X-ray absorption and emission spectroscopic measurements were carried out at beamline BL-09A at the NewSUBARU Synchrotron Radiation Facility at the University of Hyogo [9]. The sample was fixed to a copper plate holder, and its surface layer was peeled off with adhesive tape to expose a fresh surface just before it was introduced into the vacuum chamber. Absorption spectroscopy was measured using a total electron yield (TEY) method by sample current measurement and a total fluorescence yield (TFY) method by using a photodiode detector [10]. Here, the TEY method is a surfacesensitive measurement method, and the TFY method is a bulk-sensitive one.

$\mathrm{X}$-ray emission spectroscopy was carried out using a soft X-ray emission spectrometer [11] installed at the endstation of BL-09A. The light source of the excitation is a planar undulator with a horizontally polarized light with 


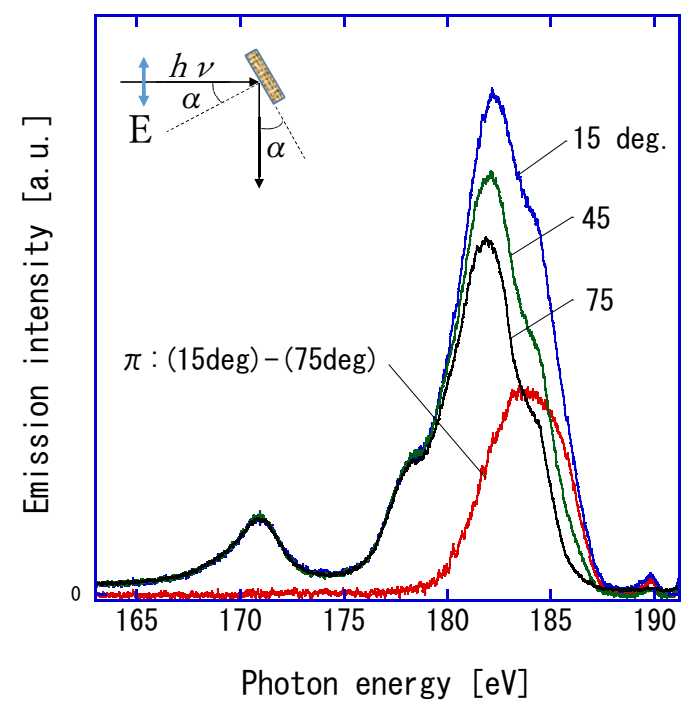

FIG. 1. Takeoff angle dependence of B-K emission spectra of h-BN powder. The red line shows the $\pi \rightarrow 1$ s component of the emission estimated by subtracting the peak shape at the takeoff angle of $75^{\circ}$ from that at the angle of $15^{\circ}$. The inset shows the relationship between the polarization direction of the incident light and the incident and takeoff angles to the sample.

a linear polarization degree of $99.3 \%$ or more [12]. The sample is placed so that the surface is along the vertical axis and can rotate around the vertical axis. Therefore, the excitation light is incident on the specimen surface with p-polarization. As shown in the inset in Fig. 1, the incident angle $\alpha$ to the sample is represented by an angle measured from the surface normal of the sample, and the takeoff angle is expressed by an angle measured from the sample surface. Since the direction in which emission light is taken into the spectrometer is fixed at $90^{\circ}$ with respect to the direction of the incident light, the values of the incident angle and the takeoff angle $\alpha$ are always equal, even when the sample is rotated around the vertical axis. The excitation energies of X-rays for the B-K and $\mathrm{C}-\mathrm{K}$ emission were $210 \mathrm{eV}$ and $310 \mathrm{eV}$, respectively; those energies were far from the absorption edges, and the excitation process was non-resonant. The energy resolution $E / \Delta E$ of the beamline spectrometer was estimated to be about 2000 in the B-K region. In addition, the resolution of the emission spectrometer was estimated to be about 1000 in the B-K region.

\section{RESULTS}

In order to confirm the performance of the soft X-ray emission spectrometer in the $\mathrm{B}-\mathrm{K}$ region, first the $\mathrm{B}$ $\mathrm{K}$ emission spectrum of hexagonal boron nitride (h-BN) powder as a standard sample was measured. Here, the powder sample we used was compacted using a press. As is well known from optical microscope observation, microcrystals of h-BN powder have a shape like a flat tile, and when pressed, they have remarkable crystal orientation and show a takeoff angle dependency of soft X-ray emission [11]. Figure 1 shows the takeoff angle dependence of the B-K emission spectra of the h-BN powder.

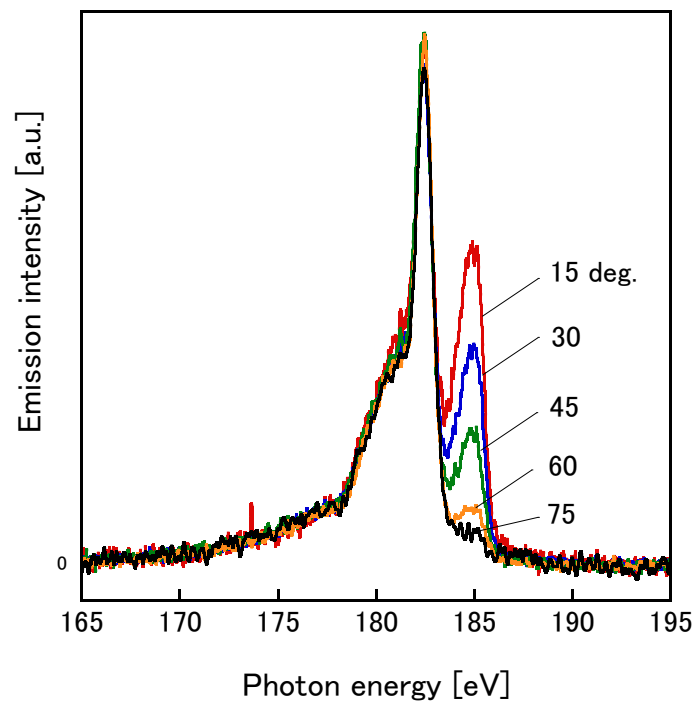

FIG. 2. Takeoff angle dependence of B-K emission spectra of trace B-doped HOPG with the excitation photon energy of $210 \mathrm{eV}$. The takeoff angle values are shown in the figure.

Here we show the spectra for three different takeoff angles: $15^{\circ}, 45^{\circ}$, and $75^{\circ}$. Since the emission intensity differs depending on the takeoff angle, the intensity was normalized so that the emission intensity at less than $178 \mathrm{eV}$ was matched. With such normalization, the peak intensity shows takeoff angle dependence at an energy greater than $178 \mathrm{eV}$ and shows a larger value as the takeoff angle becomes smaller. This is because the B-K emission spectrum is composed of two components, one derived from the lower energy side $\sigma$ orbitals and one derived from the higher energy side $\pi$ orbitals. Since the h-BN sample has a crystalline orientation, the emission intensity derived from $\pi$ orbitals changed relative to the peak intensity derived from the $\sigma$ orbitals depending on the takeoff angle.

The red curve indicated in Fig. 1 is the intensity difference obtained by subtracting the spectrum of the $75^{\circ}$ emission from the spectrum of the $15^{\circ}$ emission, and it almost reflects the shape of the $\pi$ orbital-derived component. The $\pi$ component derived is a substantially symmetrical single peak with a width of about $5 \mathrm{eV}$ centered on $184 \mathrm{eV}$. The above results are in good agreement with the results previously reported by Muramatsu et al. [13], but the takeoff angle dependence of the $\pi$ peak is somewhat larger than theirs. This seems to be due to the fact that the energy resolution of the X-ray emission spectrometer we developed [11] is higher than those used by them.

Figure 2 shows the B-K emission spectrum of the 0.32 at.\% B-doped HOPG sample. Here measurement was made at different takeoff angles: $15^{\circ}, 30^{\circ}, 45^{\circ}, 60^{\circ}$, and $75^{\circ}$. These spectra could be acquired with exposure times of 20-30 min. Since the X-ray emission intensity varies according to the takeoff angle, here it is normalized so that the intensity of the tailing portion of $170-180 \mathrm{eV}$ agrees. When normalized in this manner, the intensities of the maximum peak at $182 \mathrm{eV}$ are almost equal with respect to the takeoff angles. On the other hand, the satellite peak around $185 \mathrm{eV}$ changed according to the change in the takeoff angle, taking the minimum value at the takeoff angle of $75^{\circ}$ and increasing with the decrease 

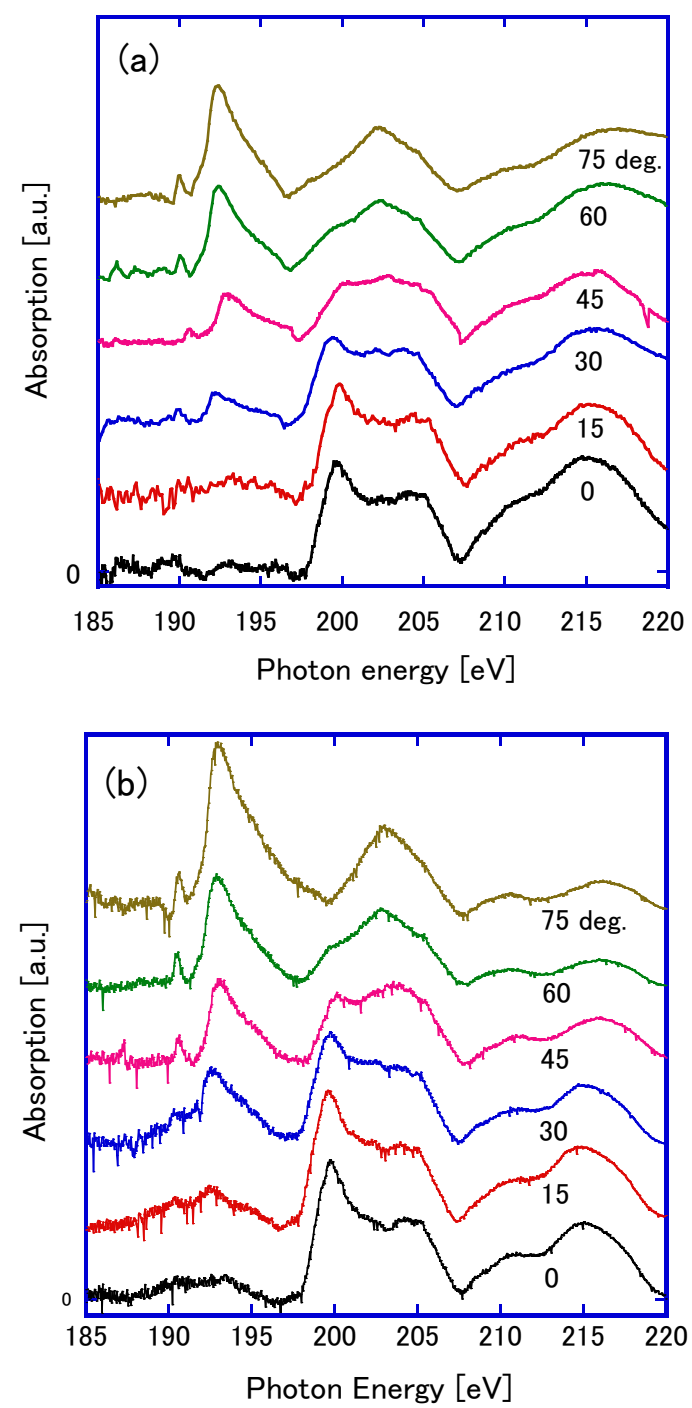

FIG. 3. Incident angle dependence of B-K absorption spectra of trace B-doped HOPG measured using (a) the TEY method and (b) the TFY method. The incident angle values are shown in the figure.

in angle. The positional relation of these peaks on the energy axis and the takeoff angle dependence of the satellite peak are very similar to those of the $\mathrm{C}-\mathrm{K}$ emission spectrum of HOPG reported previously [14]. From this, it can be inferred that the doped $\mathrm{B}$ atom is incorporated into the carbon hexagonal network structure of HOPG by replacing it with a $\mathrm{C}$ atom. Furthermore, the main peak at $182 \mathrm{eV}$ is attributed to the emission due to the transition from the $\sigma$ orbital to the B 1s state. The satellite peak at $185 \mathrm{eV}$ is attributed to emission due to the transition from the $\pi$ orbital to the B 1 s state.

Figure 3 shows the B-K absorption spectrum of the 0.32 at.\% B-doped HOPG sample for six angles of incidence: $0^{\circ}, 15^{\circ}, 30^{\circ}, 45^{\circ}, 60^{\circ}$, and $75^{\circ}$. Figures $3(\mathrm{a})$ and $3(\mathrm{~b})$ are measured by the TEY method and the TFY method, respectively. The spectra measured by the TEY method and the TFY method are very similar. This indicates that the electronic state of the sample surface and the bulk has not changed greatly. These peak shapes were very different from the peak shape of the B-K absorption of B-doped HOGF reported by Hanafusa et al. [7]. They

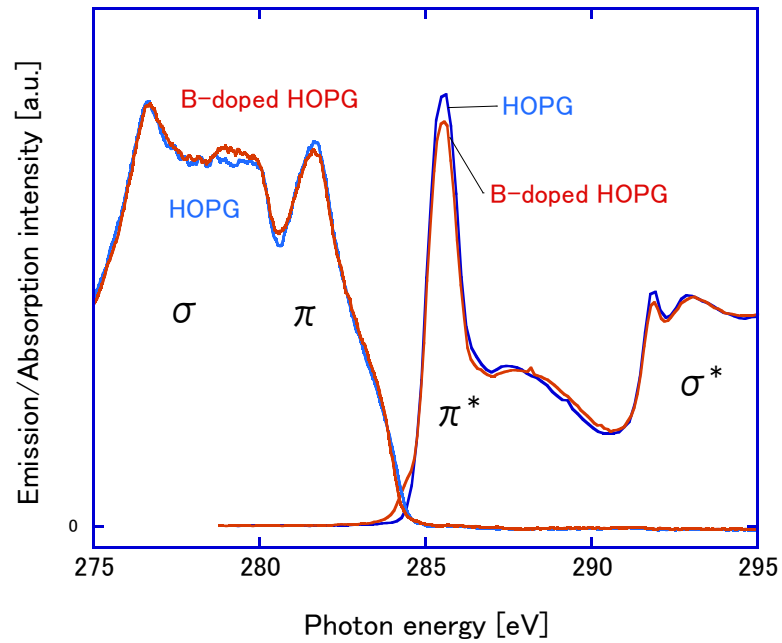

FIG. 4. Soft X-ray emission and absorption spectra in the vicinity of the $\mathrm{C}-\mathrm{K}$ absorption edge of B-doped HOPG (red curves) and undoped HOPG (blue curves) measured at the incident/takeoff angle of $45^{\circ}$.

were rather similar to that of HOPG doped with B and N reported by Shimoyama et al. [15]. When normalized so that the spectral intensities around $202 \mathrm{eV}$ become equal, the peak intensity around $192 \mathrm{eV}$ monotonically increases with the increasing incident angle. The positional relation of these peaks on the energy axis and their incident angle dependence are very similar to those of the C-K absorption spectra of HOPG [16]. Based on this, the peak around $192 \mathrm{eV}$ is attributed to absorption by transition from B 1 s to the $\pi^{*}$ orbital. Additionally, a number of peaks at $200 \mathrm{eV}$ or greater are attributed to absorption by the transition from $\mathrm{B} 1 \mathrm{~s}$ to the $\sigma^{*}$ orbital.

As comparing to the incident angle dependence of $\pi^{*}$ peak in Fig. 3 to the takeoff angle dependence of $\pi$ peak in Fig. 2, the dependency of the angle may seems opposite. However, the relation between X-ray polarization and the symmetry of $\pi / \pi^{*}$ orbital is essentially the same between emission and absorption under the dipole transition regime. Seemingly opposite angular dependence is basically due to the configuration of the beamline and the emission spectrometer as explained in the experimental section. The large signal of emissions from $\pi$ orbital observed at small takeoff angle $\alpha$ can be explained by the electric vector of emission which is perpendicular to the sample surface due to crystalline structure of HOPG. For $\mathrm{X}$-ray absorption, small angle $\alpha$ is corresponding to near normal incidence, hence absorption of $\pi^{*}$ is suppressed.

Figure 4 shows the soft X-ray emission and absorption spectra in the vicinity of the C-K absorption edge of Bdoped HOPG and undoped HOPG measured at the incident/takeoff angle of $45^{\circ}$. These peak intensities were normalized so that the base emission intensity around $277 \mathrm{eV}$ and the base absorption intensity around $293 \mathrm{eV}$ were equal. As is well known, the X-ray emission intensity reflects the density of states of the valence band in solids. Furthermore, the X-ray absorption intensity reflects the density of states of the conduction band in solids. Although the spectra of B-doped HOPG and undoped HOPG are very similar, there is a slight increase and decrease in the conduction band density of states at 


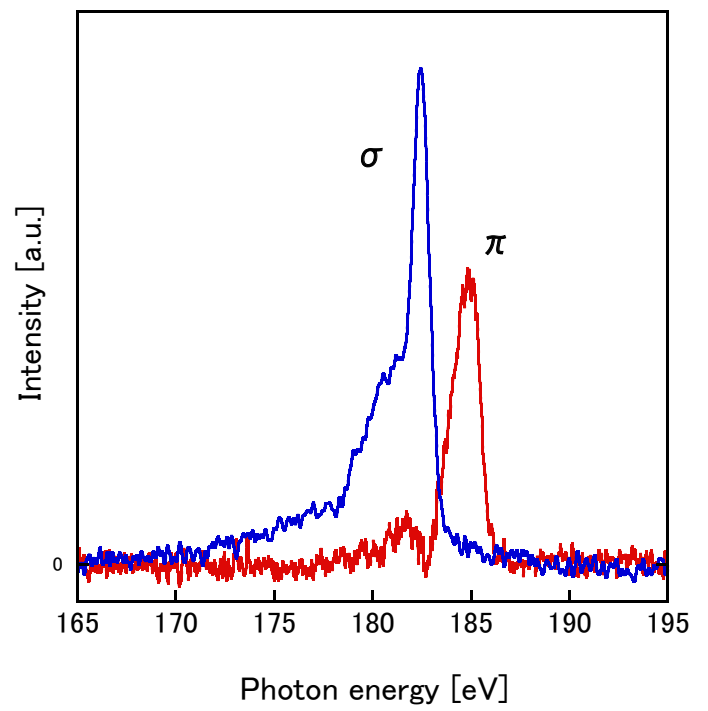

FIG. 5. Peak distillation of the $\pi$ and $\sigma$ components of the $\mathrm{B}-\mathrm{K}$ emission spectrum of the B-doped HOPG sample.

the tailing of the absorption edge near $284 \mathrm{eV}$ and at the $\pi^{*}$ peak of $285.4 \mathrm{eV}$, respectively, for the B-doped HOPG.

\section{DISCUSSION}

Figure 5 shows the results of peak distillation of the $\pi$ and $\sigma$ components of the B-K emission spectrum of the B-doped HOPG sample. In the process of this peak distillation, first, the $75^{\circ}$ spectrum with a small $\pi$ contribution was subtracted from the spectrum of the takeoff angle of $15^{\circ}$, which contributes greatly to the satellite peak of $185 \mathrm{eV}$, to obtain the approximate shape of the $\pi$ peak. Next, the $\sigma$ peak shape was obtained by multiplying the functional form by the magnification coefficient so that the $\pi$ peak intensity at the takeoff angle of $15^{\circ}$ coincided with the original spectrum. From this result, it was found that the $\pi$ component around $185 \mathrm{eV}$ of the X-ray emission spectrum is represented by a single, almost Gaussian shape, and the full width at half maximum (FWHM) of the $\pi$ peak is about $1.7 \mathrm{eV}$. As shown in the previous section, this peak intensity increases as the takeoff angle decreases. On the other hand, the FWHM of the main $\sigma$ peak at $182 \mathrm{eV}$ is about $1.2 \mathrm{eV}$.

In a previous study, Hanafusa et al. reported B-K emission spectra for a high-temperature synthesized B-doped HOGF [7]. According to their quantum chemical consideration, both the main peak of $182 \mathrm{eV}$ and the high-energy satellite peak of $185 \mathrm{eV}$ are attributed to $\pi$ orbitals, and only the tailing on the low energy side is attributed to $\sigma$ orbitals. However, from the measurement of the takeoff angle dependence in this study, it was found that it is more appropriate to think that the main peak at $182 \mathrm{eV}$ is derived from the $\sigma$ orbital.

As described in the experimental section, the excitation energy of our XES measurement is a non-resonant condition. Hence, there is no incident angle dependence due to the polarization of the excitation X-rays. In the case of non-resonant excitation, only the dependence of the XES on the observation direction from the sample is observed regardless of the configuration of the excita-

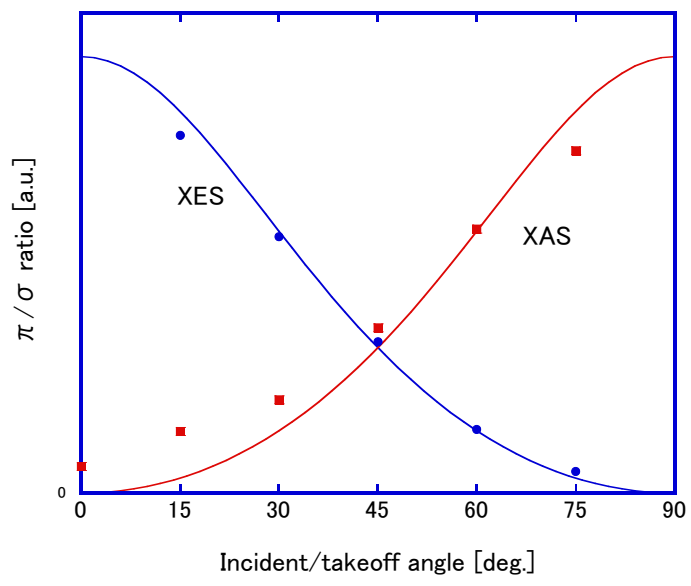

FIG. 6. Incident/takeoff angle dependence of the $\sigma$ and $\pi$ peak intensity ratio $(\sigma / \pi)$ of B-K emission and absorption spectrum of trace B-doped HOPG. The lines denote the theoretical values calculated using Eqs. (2) and (3).

tion X-ray beam. The takeoff angle dependence of the soft X-ray emission spectrum of HOPG laminated with a hexagonal network structure is theoretically explained by considering the orientation of $\sigma$ and $\pi$ orbitals. According to Mansour et al. [17], the takeoff angle dependence of the total emission intensity, $I(E, \alpha)$, is calculated by adding together the contributions of the $\sigma$ component and the $\pi$ component as follows:

$$
I(E, \alpha)=I_{\sigma}(E) \frac{1+\sin ^{2} \alpha}{2}+I_{\pi}(E) \cos ^{2} \alpha
$$

From this, the emission intensity ratio of the $\pi$ and $\sigma$ components, $\{I(\pi) / I(\sigma)\}_{\mathrm{em}}$, is

$$
\left\{\frac{I(\pi)}{I(\sigma)}\right\}_{\mathrm{em}}=\frac{I_{\pi}(E) \cos ^{2} \alpha}{I_{\sigma}(E) \frac{1+\sin ^{2} \alpha}{2}}=K \frac{\cos ^{2} \alpha}{1+\sin ^{2} \alpha}
$$

Here, $K$ is an arbitrary constant. With a similar consideration [16], the absorption intensity ratio of $\pi$ and $\sigma$ components, $\{I(\pi) / I(\sigma)\}_{\text {abs }}$, is

$$
\left\{\frac{I(\pi)}{I(\sigma)}\right\}_{\mathrm{abs}}=\frac{I_{\pi}(E) \sin ^{2} \alpha}{I_{\sigma}(E) \frac{1+\cos ^{2} \alpha}{2}}=K \frac{\sin ^{2} \alpha}{1+\cos ^{2} \alpha}
$$

Figure 6 shows the incident/takeoff angle dependence of $\{I(\pi) / I(\sigma)\}$ values calculated for the measured emission and absorption spectra using peak values. It can be seen that the values of $\{I(\pi) / I(\sigma)\}$ at each incident/takeoff angle fit well with the theoretical calculation curve using Eqs. (2) and (3). As a result, the doped boron atom substitutes for the $\mathrm{C}$ atom constituting HOPG and enters the $\mathrm{C}$ site; consequently, it has an $\mathrm{sp}^{2}$ type hybridized orbital similar to the $\mathrm{C}$ electron and $\{I(\pi) / I(\sigma)\}$ ratio on the incident/takeoff angle dependence.

\section{CONCLUSION}

We could successfully observe the soft X-ray absorption and emission spectra near the B-K absorption edge of $\mathrm{B}$ atoms for trace $(0.32 \%)$ boron-doped HOPG, including 
changes in incident and takeoff angles. We showed more clearly from the spectroscopic viewpoint that the B atom entered the site where the $\mathrm{C}$ atom was substituted. Based on analysis of the dependence of the absorption and emission spectra on the incident/takeoff angle, it is reasonable to think that the main peak around $182 \mathrm{eV}$, which is derived from the $\pi$ orbital according to the quantum chemical calculation in the preceding report, is derived from the $\sigma$ orbital. We also measured the C-K emission and absorption spectra of the above sample and found that the density of states of the absorption edge tailing slightly increases with boron doping.

\section{ACKNOWLEDGMENTS}

We would like to thank Dr. Tadao Iwata, formerly of the Japan Atomic Energy Research Institute (JAERI), for offering samples of B-doped HOPG and his participation in many useful discussions.
[1] J. Stöhr, NEXAFS Spectroscopy (Springer, Berlin, 2003).

[2] U. Bergmann, and P. Glatzel, Photosynth. Res. 102, 225 (2009).

[3] C. E. Lowell, J. Am. Ceram. Soc. 50, 142 (1967).

[4] Y. Wang, D. C. Alsmeyer, and R. L. McCreery, Chem. Mater. 2, 557 (1990).

[5] M. Endo, T. Hayashi, S.-H. Hong, T. Enoki, and M. S. Dresselhaus, J. Appl. Phys. 90, 5670 (2001).

[6] M. O. Krause, J. Phys. Chem. Ref. Data 8, 307 (1979).

[7] A. Hanafusa, Y. Muramatsu, Y. Kaburagi, A. Yoshida, Y. Hishiyama, W. Yang, J. D. Denlinger, and E. M. Gullikson, J. Appl. Phys. 110, 053504 (2011).

[8] E. Yagi, T. Iwata, T. Urai, and K. Ogiwara, J. Nucl. Mater. 334, 9 (2004).

[9] M. Niibe, M. Mukai, S. Miyamoto, Y. Shoji, S. Hashimoto, A. Ando, T. Tanaka, M. Miyai, and H. Kitamura, AIP Conf. Proc. 705, 576 (2004).
[10] M. Niibe, T. Kotaka, and T. Mitamura, J. Phys.: Conf. Ser. 425, 132008 (2013).

[11] M. Niibe and T. Tokushima, AIP Conf. Proc. 1741, 030042 (2016).

[12] M. Niibe, M. Mukai, H. Kimura, and Y. Shoji, AIP Conf. Proc. 705, 243 (2004).

[13] Y. Muramatsu, E. M. Gullikson, and R. C. C. Perera, Adv. X-ray Chem. Anal. Japan 34, 153 (2003) (in Japanese).

[14] M. Niibe, T. Tokushima, N. Takehira, and Y. Araki, J. Electron Spectrosc. Relat. Phenom. 220, 118 (2017).

[15] I. Shimoyama, Y. Baba, T. Sekiguchi, M. N. Uddin, and M. Nagano, Photon Factory Activity Report 26B, 97 (2009).

[16] R. A. Rosenberg, P. J. Love, and V. Rehn, Phys. Rev. B 33, 4034 (1986).

[17] A. Mansour, S. E. Schnatterly, and R. D. Carson, Phys. Rev. B 31, 6521 (1985). 